\title{
Sarcoidosis presenting with multiple tattoo granulomata
}

\author{
J. M. I. IVESON \\ M.B., M.R.C.P.
}

\author{
J. A. CotTerill \\ B.Sc., M.D., M.R.C.P.
}

\author{
V. WRIGHT \\ M.D., F.R.C.P.
}

A patient with sarcoidosis, multiple tattoo granulomata and arthritis is described. A combination of tattoo granulomata unrelated to any one colour in the tattoo and granulomatous inflammation elsewhere is indicative of sarcoidosis.

\section{Introduction}

The presenting features of sarcoidosis may be very varied, reflecting the multi-system involvement characteristic of this disease. We describe an unusual manifestation of sarcoidosis in a tattooed patient who developed multiple nodules, not limited to any one colour in his many tattoos.

\section{Case report}

The patient, a 32-year-old male unemployed gardener, had been tattooed whilst in the Army some 13 years previously. For the last 18 months he had noticed non-itchy lumps appearing in many of the tattoos, together with a swelling of the left ring finger, right thumb, left third toe and right second toe. More recently he had also developed swelling behind the left knee.

On examination, the salient clinical features were multiple granulomata present in many differing tattoos. These granulomata were observed in brown (iron), green (chromium) and red (mercury) areas of different tattoos (Fig. 1). He also showed fusiform swelling of the right thumb, left ring finger, left third and right second toes (Fig. 2). There was also an effusion of the left knee with a popliteal cyst.

Investigations showed a normal full blood count, urea, electrolytes, uric acid, serum proteins and liver function tests. The RA latex, DAT and ANF were negative. The Mantoux reaction was negative down to the dilution of $1 / 100$. Chest X-ray showed bilateral hilar lymphadenopathy. X-rays of hands and feet showed abnormal trabeculation with cysts in the phalanges of the affected fingers in keeping with a diagnosis of sarcoid osteitis. Twenty millilitres of turbid yellow fluid was aspirated from the left knee. This contained 400 red cells $/ \mathrm{mm}^{3}$ and 220 white cells,

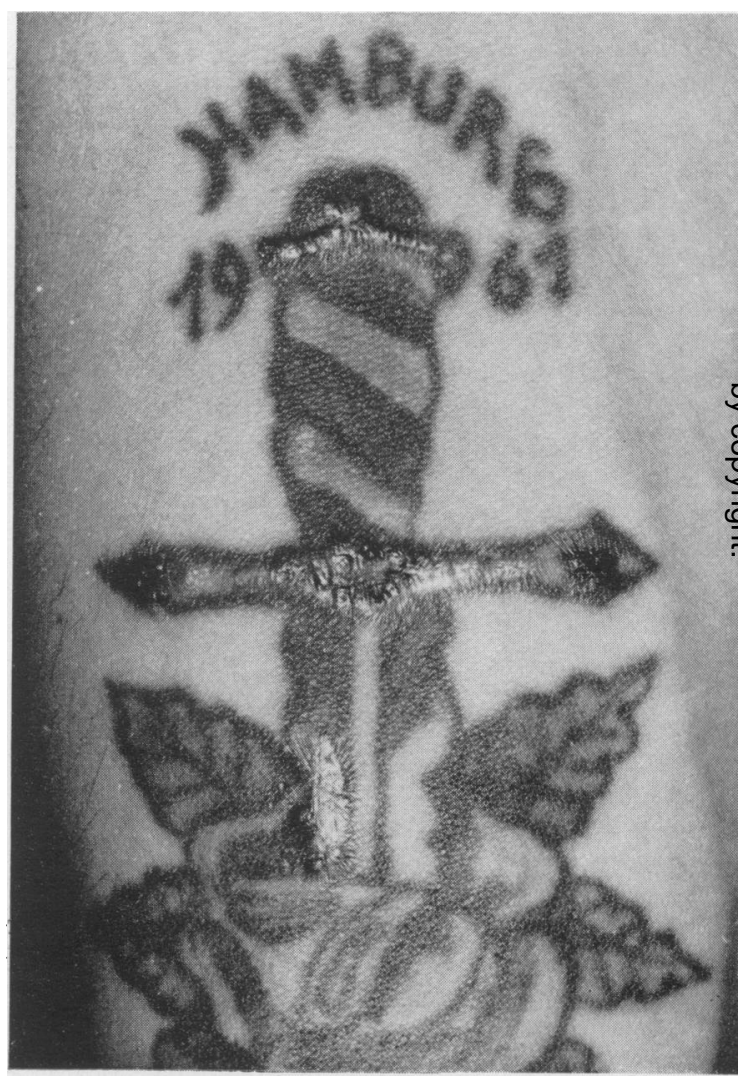

FIG. 1.

$85 \%$ of which were polymorphonuclear leucocytes. Patch tests to nickel, cobalt, chromium and mercury were all negative after 48 and $96 \mathrm{hr}$.

\section{Histological features}

A biopsy of the swelling of the right thumb was characteristic of sarcoidosis and a biopsy of a skin tattoo nodule demonstrated a dermis containing pigment and a severe granulomatous inflammatory 


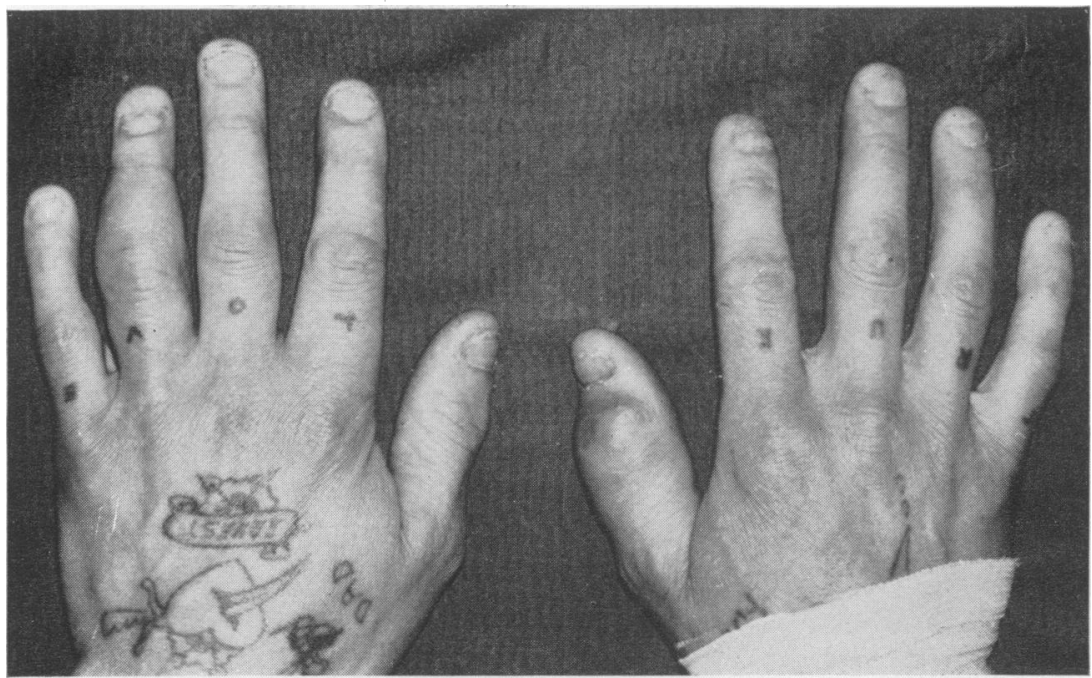

reaction with giant cells and histiocytes. The appearances were felt to be compatible with either sarcoidosis or a reaction to foreign material.

\section{Comment}

Although, radiologically, sarcoid osteitis occurs in $17 \%$ of cases of sarcoidosis (Pygott, 1970), it is the presenting clinical feature of the disease much less frequently. When present, it is frequently associated with skin involvement, especially of the more indolent and chronic forms such as lupus pernio (Scadding, 1967). Moreover, sarcoidosis presenting in scars may be the only cutaneous clue to generalized sarcoidosis (James, 1959).

There are many complications of tattooing (Beerman and Lane, 1954; Scutt, 1972). These range from simple infection at the site, transmission of infection, e.g. syphilis, tuberculosis and hepatitis, to allergic reactions to the pigments used, usually mercury (Scutt, 1972). 'Sarcoid' reactions due to pigment are not uncommon but the development of multiple tattoo granulomata as a presenting feature of sarcoidosis is rare. We can only find seven such case reports in the world literature (Lubeck and Epstein, 1952; Obermayer and Hassen, 1955; Weidman, Andrade and Franks, 1966; Dickinson, 1969; Scutt, 1972). Rorsman et al. (1969) described three patients who developed tattoo granulomata and uveitis simultaneously. However, in contrast to our patient, these granulomata were seen only in light blue areas of the tattoo which were probably coloured with cobaltous aluminate and excision of the inflamed tattoos was followed by improvement of the uveitis in two of three patients. There was no definite evidence that these patients had sarcoidosis and the Kveim test was not done.

Are the tattoos in our patient in any way relatedo to the development of his sarcoidosis? This seems unlikely but it may be that a combination of previous trauma and pigment determined the distribution of the sarcoid lesions in the tattoos. Moreover, although two of the three cases of Rorsman et al. (1969) had positive patch tests to cobalt, our patient did not show positive patch tests to any of the metals present in the tattoos. We would conclude that our patient's tattoos determined the site of his cutaneous sarcoidosis but were not related to the pathogenesis of this condition.

A combination of the tattoo granulomata not limited to any one colour with granulomatous inflammation elsewhere is indicative of generalized sarcoidosis.

\section{Acknowledgment}

Mrs V. M. Dickinson, who kindly typed the manuscript.

\section{References}

Beerman, H. \& LANe, R.A.G. (1954) Tattoo. American Journal of Medical Science, 277, 444.

Dickinson, J.A. (1969) Sarcoidal reactions in tattoos. Archives of Dermatology, 100, 315.

JAMES, D.G. (1959) Dermatological aspects of sarcoidosis. Quarterly Journal of Medicine, 28, 109.

LUBECK, G. \& EPSTEIN, E. (1952) Complications of tattooing. California Medicine, 67, 83.

Obermeyer, M.E. \& HASSEN, M. (1955) Sarcoidosis with sarcoidal reaction in tattoo. Archives of Dermatology, 71 , 766.

Pygotr, F. (1970) Sarcoidosis in bone. Postgraduate Medical Journal, 46, 505. 
Rorsman, H., Dahlquist, I., Jacobsson, S., BrehmerAnderson, E., Ehinger, B., Linell, F. \& Rorsman, G. (1969) Tattoo granuloma and uveitis. Lancet, ii, 27.

Scadding, J.G. (1967) Sarcoidosis, p. 208. Eyre \& Spottiswood, London.
Scutr, R.W.B. (1972) The medical hazards of tattooing. Hospital Medicine, 2, 195.

Weidman, A.I., ANDrade, R. \& Franks, A.G. (1966) Sarcoidosis. Archives of Dermatology, 97, 320.

\section{Multiple carcinomas of the large and small bowel in childhood}

\author{
DAVID R. HARVEY \\ M.B., B.S., L.R.C.P., F.R.C.S.
}

\author{
Fernando J. Paradinas \\ L.R.C.P., L.R.C.S., M.R.C.Path.
}

\begin{abstract}
Summary
A case is reported of multiple metachronous carcinomas of the small and large intestine in a child. The latter were associated with multiple adenomatous polyps in the colon. A desmoid tumour and multiple keloids developed in the laparotomy scars. The relationship of this case to Gardner's syndrome and polyposis coli is discussed and the literature on intestinal carcinoma in childhood is briefly reviewed.
\end{abstract}

\section{Introduction}

Small bowel carcinoma has occasionally been described in association with Gardner's syndrome and large bowel carcinoma is a common late complication of this syndrome and of polyposis coli. However, we have not found previous reports of multiple small and large bowel carcinomas in this age group.

\section{Case report}

A 13-year-old girl was admitted in October 1970, with weight loss, abdominal pain, anaemia, fever and a mass in the left iliac fossa. She and two siblings had had idiopathic thrombocytopenic purpura which in her case had remitted spontaneously. At laparotomy, an ulcerating carcinoma of the lower descending colon and an adenomatous polyp found $5 \mathrm{~cm}$ distal to the carcinoma were removed by left hemicolectomy. The carcinoma was of mucoid type (Fig. 1) and involved the full thickness of the wall and one paracolic lymph node. Post-operative recovery was uneventful and extensive enquiries revealed no family history of polyposis coli or carcinoma of the colon. Follow-up barium studies revealed no further polyps.

In August 1971, she was readmitted with acute on

Reprint requests to: Dr D. R. Harvey, Charing Cross Hospital, Fulham Palace Road, London W6 8RF. subacute intestinal obstruction and a mass in the right iliac fossa. At laparotomy two small bowel carcinomas were resected: one in the upper jejunum, $7.5 \mathrm{~cm}$ distal to the duodenal jejunal flexure, and one $10 \mathrm{~cm}$ proximal to the ileocaecal junction. Microscopy showed non-mucoid adenocarcinomas involving the submucosa and inner muscle only (Fig. 2)? This supported the operative impression of two separate primary small intestinal adenocarcinomas rather than metastases from the original mucoidi carcinoma. Lymph nodes taken from the root of the mesentery showed marked follicular hyperplasia, but no evidence of metastases.

The patient was again admitted in April 1972 with a mass in the original colectomy scar. Clinically this was thought to be a recurrence of the carcinoma, but at operation and on subsequent histology it proved to be a desmoid tumour which was widely resected as a monobloc procedure. Reconstitution of the anterior abdominal wall was by primary suture with minimal undermining. During follow-up in 1972 repeated chest $\mathrm{X}$-rays, a barium meal with follow through, a barium enema and sigmoidoscopy at 6-monthly intervals were all normal. In particular there was no evidence of further intestinal polyps.

In September 1973 the patient attended again complaining of lassitude, diarrhoea and headache. Anaemia and a mobile tumour palpable in the left iliac fossa were the clinical findings. Biopsy of a large pedunculated tumour of the rectum showed part of an adenomatous polyp. A barium enema showed multiple colonic polyps and a stricture of the sigmoid (Fig. 3). A radiological diagnosis of carcinoma was confirmed at operation. The colon showed an ulcerated stenosing tumour, merging with a sessile polyp immediately above the ulcer (Fig. 5). Up to fourteen other polyps were present in the total colectomy specimen (Figs. 4 and 5) and two further 